\title{
EchoGéo
}

$28 \mid 2014$

Police : les espaces de l'ordre, l'ordre en espace

\section{Ordre social, ordre spatial : territorialisation des politiques de sécurité publique à Rio de Janeiro}

Justine Ninnin

\section{OpenEdition}

1 Journals

Édition électronique

URL : https://journals.openedition.org/echogeo/13794

DOI : 10.4000/echogeo. 13794

ISSN : 1963-1197

Éditeur

Pôle de recherche pour l'organisation et la diffusion de l'information géographique (CNRS UMR 8586)

Référence électronique

Justine Ninnin, «Ordre social, ordre spatial : territorialisation des politiques de sécurité publique à Rio de Janeiro », EchoGéo [En ligne], 28 | 2014, mis en ligne le 08 juillet 2014, consulté le 31 juillet 2021.

URL : http://journals.openedition.org/echogeo/13794; DOI : https://doi.org/10.4000/echogeo.13794

Ce document a été généré automatiquement le 31 juillet 2021.

EchoGéo est mis à disposition selon les termes de la licence Creative Commons Attribution - Pas d'Utilisation Commerciale - Pas de Modification 4.0 International (CC BY-NC-ND) 


\title{
Ordre social, ordre spatial : territorialisation des politiques de sécurité publique à Rio de Janeiro
}

\author{
Justine Ninnin
}

\section{Introduction}

1 Face à une violence urbaine croissante, une concentration des pouvoirs par des groupes criminels et une légitimité policière ébranlée, la thématique de la sécurité publique est devenue incontournable dans les débats publics au Brésil. Un défi qui se pose d'autant plus dans le contexte d'accueil de méga-événements comme la Coupe du Monde de football de 2014 et les Jeux Olympiques de 2016. L'imaginaire social projette sur les favelas les stigmatisations de la marginalité et du désordre urbain, la tendance à la superposition de la carte de la violence à celle de la pauvreté conduit les pouvoirs publics à multiplier les politiques territorialisées dans les favelas visant à rétablir leur emprise sur ces espaces. C'est dans ce contexte d'articulation entre contrôle social et contrôle spatial que nous nous intéressons aux politiques de sécurisation à Rio de Janeiro. En identifiant les territoires d'action ciblés par ces politiques, nous cherchons à analyser la production socio-spatiale de l'ordre. Qui sont les acteurs spatialisés générateurs de contrôle et d'ordre? Comment se matérialise la politique de sécurité publique sur ce territoire contrasté ? Quels sont les choix et méthodes appliqués par les institutions policières?

2 La révision de la stratégie de sécurité publique se matérialise, en 2008, par la politique de «pacification » élaborée par le Secrétariat de Sécurité Publique de l'État de Rio de Janeiro. Ce processus vise dans un premier temps à reprendre le contrôle des territoires dominés par des criminels, puis à revaloriser les rapports entre la population et les policiers en mettant en place une police communautaire localisée aux endroits stratégiques des favelas qui prend le nom d'Unité de Police de Pacification (UPP) à Rio de Janeiro. La sécurité est affirmée comme droit fondamental du ressort de la sphère 
publique et elle se renforce par des projets de développement socio-économique regroupés dans le programme «UPP social» de la mairie, ayant pour prérogative la participation de la société civile et des habitants dans l'action et le débat public.

3 Cet article vise, dans un premier temps, à mieux comprendre les territoires sur lesquels s'appliquent les interventions policières de pacification. Après avoir souligné les différents types de pouvoirs qui entrent en conflit dans les favelas, nous chercherons à confronter les espaces de concentration d'actes criminels aux espaces d'insécurité perçue. Enfin nous analyserons l'impact de la police de proximité sur l'ordre sociospatial. Ce texte s'appuie sur une approche empirique qui mobilise des observations et des entretiens sur le terrain, plus spécifiquement dans deux favelas pacifiées depuis 2011 situées dans la zone sud de Rio de Janeiro : Vidigal et Rocinha ${ }^{1}$ (cf. illustrations ${ }^{\circ} 1$ et 2). La première compte environ 12000 habitants et la deuxième, souvent présentée comme l'une des plus grandes favelas d'Amérique Latine, environ 100000.

Illustration 1 - Pacification et découpage des zones à Rio de Janeiro

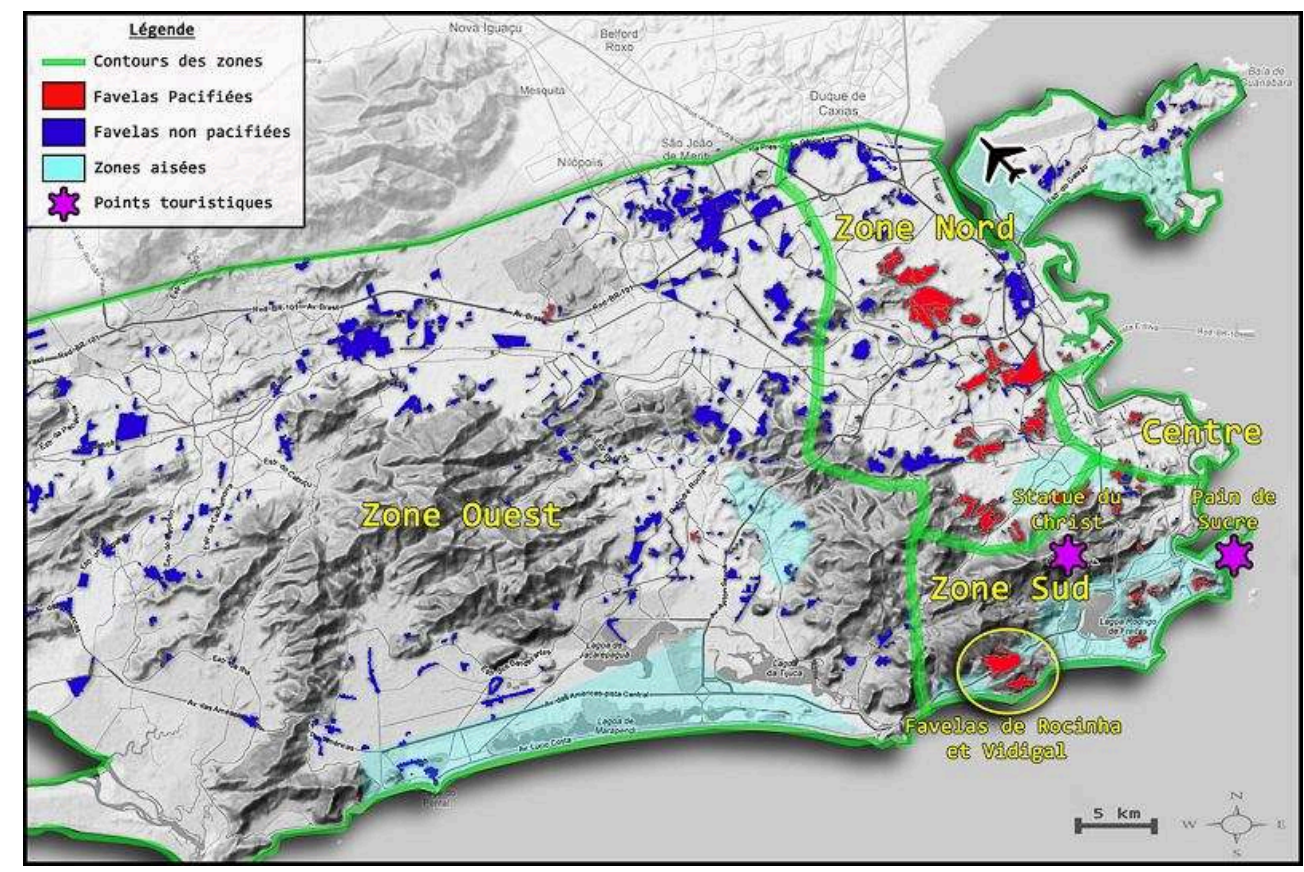

Source : UPP social ; réalisation : J. Ninnin. 
Illustration 2 - Zoom sur les favelas de Rocinha et de Vidigal dans la zone sud de Rio de Janeiro

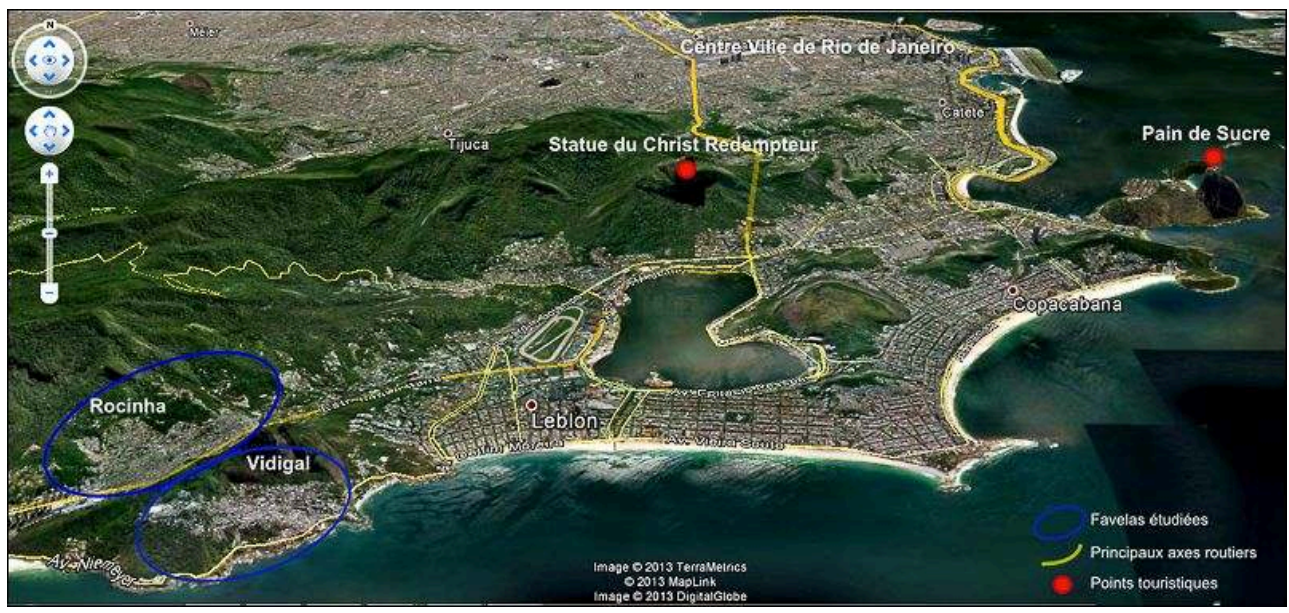

Source : Google Earth.

\section{Mieux comprendre le territoire d'intervention : une police spécifique pour un espace singulier?}

\section{Une approche locale de la question de la sécurité}

4 Depuis plusieurs décennies la ville de Rio de Janeiro ne cessait de voir ces indices criminels augmenter. Toutefois, concernant les premiers signaux de diminution se remarquent ces dernières années.

Illustration 3 - Taux d'Homicides entre 1980 et 2010 (pour 100000 habitants)

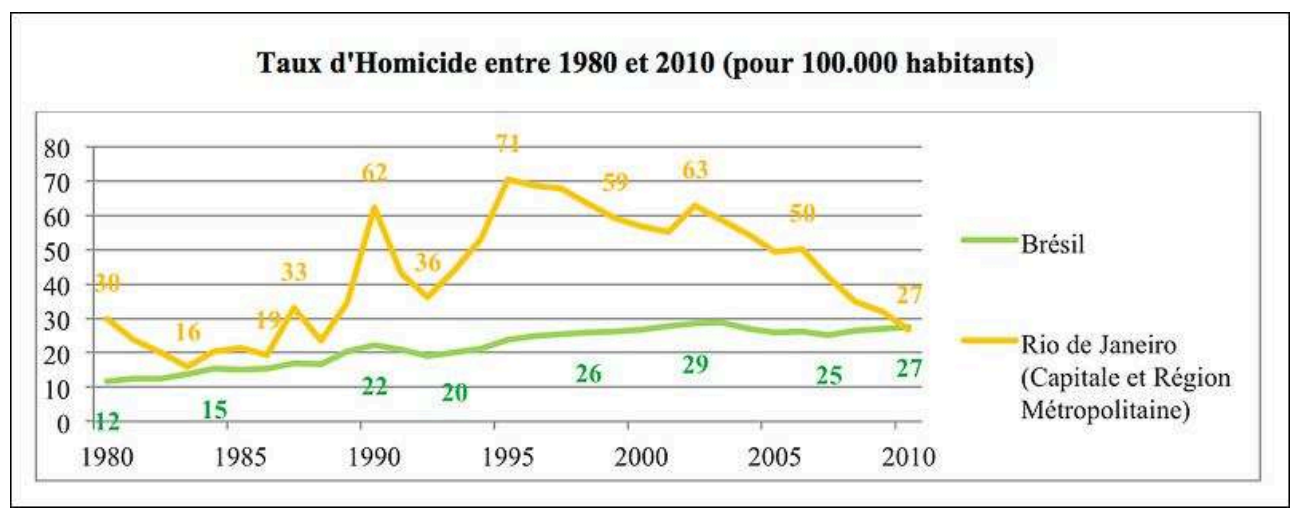

Sources: SIM/SVS/MS (Waiselfisz, 2013)

Différentes expériences ont tenté de combattre l'insécurité ; cependant, jusqu'en 2008, la police n'était présente dans les favelas que lors d'opérations ponctuelles. On peut, entre autres, citer comme tentatives avortées, le projet de Centres Communautaires de Défense de la Citoyenneté dans les années 1980, le programme "Operação Rio" d'occupation militaire des favelas en 1994, le projet «Mutirão da Paz» de 1999 ou encore les GPAE (Groupe de Police dans les Zones Spéciales) en 2000 (Leeds, 1998). C'est en 2008, que la mairie tente à nouveau l'expérience d'occupation de favelas par les forces armées avec la pacification. 
6 Pacifier une favela, c'est tout d'abord envoyer des policiers et chars blindés dans ces lieux où les autorités publiques ont perdu leur emprise afin d'appréhender les armes, la drogue et les hommes ayant des mandats de prison. A Rocinha et Vidigal, l'opération Choque de Paz (Choc de paix) a débuté dans la nuit du 13 novembre 2011. Près de 1500 hommes (des policiers militaires dont le Bataillon des Opérations Spéciales de Police: BOPE et le Bataillon de Police de Choc, des marines, policiers fédéraux et policiers civils) et une dizaine de blindés ont pénétré dans ces favelas, survolées par des hélicoptères. En une demi-journée les forces de l'ordre les "dominèrent " comme l'illustre la cérémonie finale de hissement des drapeaux du Brésil et de l'État de Rio de Janeiro. Ces opérations ce sont déroulées relativement pacifiquement, sans tirs, la population ayant été informée de la date par la municipalité. Certains trafiquants ont été arrêtés les jours précédents et notamment le chef de Rocinha; d'autres ont fui. C'est l'implantation d'UPP sur le long terme venant prendre le relais du BOPE quelques mois plus tard qui différencie cette opération des expériences d'occupation passées. Avec un effectif de 700 policiers, les UPP ont été inaugurées en janvier 2012 à Vidigal et en septembre 2012 à Rocinha et sont renforcées par la suite par les programmes de l'UPP social en partenariat avec la société civile. Ces programmes sont spécifiques à chaque favela en fonction de leur nécessité. Dans une volonté de rompre avec les politiques de sécurité autoritaires héritées de la dictature, mobilisant notamment une police extrêmement répressive, ces nouvelles orientations différencient les politiques de sécurité publique des politiques publiques de sécurité en associant diverses institutions et actions politiques pouvant avoir un impact sur les problèmes de violence. Il s'agit, en d'autres termes, d'allier la sécurité physique à la sécurité citoyenne en combinant la "policiarisation» à d'autres actions publiques capables de garantir les droits fondamentaux. On s'oriente vers une territorialisation de l'action sécuritaire et sociale, c'est-à-dire que les programmes s'ancrent dans une approche locale en cherchant à prendre en compte la singularité socio-spatiale des lieux d'action.

\section{Les favelas : un espace marginalisé ?}

7 Pendant près d'un siècle, les favelas ont accueilli des populations qui ne trouvaient pas de toit dans une ville en proie à la crise du logement à l'époque de l'accélération de l'industrialisation et de l'urbanisation. Elles sont rapidement devenues un "problème urbain", l'opinion publique les associant aux notions de désordre, de précarité, d'insalubrité ou encore de marginalité. Jusque dans les années 1970, les autorités publiques ont prôné leur éradication. Avec la prolifération des groupes criminels armés (GCA), l'expansion du narcotrafic ou encore l'augmentation des violences urbaines, dans les années 1980, les représentations sociales associent naturellement pauvreté, criminalité, insécurité et favela. Les médias contribuent à en véhiculer un symbolisme négatif à travers la fascination télévisuelle et la mise en spectacle de la violence.

Pour contrecarrer ces représentations stigmatisantes et donner une valeur emblématique à ces espaces, l'utilisation du terme de communauté s'étend. Une notion qui toutefois tend à dissimuler les inégalités en mettant en évidence des ressemblances internes présumées « culturelles ». Or, selon Licia Valladares, il n’y a « ni homogénéité, ni spécificité, ni unité entre elles, ni même en leur sein pour les grandes » (Valladares, 2006, p. 161). Loin de vivre dans des enclaves de la ville fonctionnant de manière isolée et autonome, une grande partie des habitants est insérée dans le tissu urbain. Des transformations significatives sont apparues que ce soit notamment dans les modes 
d'occupation, les infrastructures ou les équipements. Auparavant essentiellement spontanées et précaires les favelas ont rapidement connu une complexification du bâti avec l'apparition d'immeubles et de résidences plus luxueuses, tout en se maintenant au cœur des enjeux de contrôle du territoire. Longtemps, les pouvoirs publics n'y étaient présents que de manière incomplète ou insuffisante, aussi d'autres groupes de pouvoirs en ont profité pour prendre le contrôle par la force.

\section{Un ordre socio-spatial régi par des entités privées}

\section{Différents types de pouvoirs spatialisés}

9 On peut identifier trois principales activités criminelles à Rio de Janeiro : le Jogo de Bicho (loteries clandestines), les trafiquants de drogue et les milices. Jusque dans les années 1980, les banqueiros, les propriétaires du Jogo de Bicho, forment, selon Michel Misse, "l'activité criminelle la plus proche d'une organisation de type mafieuse au Brésil » (Misse, 2011, p. 15). Ce sociologue parle de "réseaux » se disputant le contrôle des territoires de jeu et même d'«institution informelle » hiérarchisée ayant comme caractéristiques une solide adhésion des classes populaires, une force politique ainsi qu'une interconnexion avec la police. Les banqueiros multipliaient les responsabilités dans les favelas qu'ils "géraient» (présidents d'école de samba, de club de foot, contribution à la vie locale sous forme d'associations ou d'investissements, etc.).

A partir des années 1960, durant la dictature militaire, émerge un autre type d'organisation criminelle: les factions. Autrefois le trafic de drogue s'organisait essentiellement autour de réseaux de vente de cannabis, puis, à partir des années 1970, le profil du trafic évolue avec l'introduction de la cocaïne. Différentes factions tentent alors de prendre le contrôle des favelas (le CV : Comando Vermelho, la ADA : Amigos dos amigos et le TC: Terceiro Comando). Selon Michel Misse, 10 à $15 \%$ de la population de la ville vit dans des zones contrôlées par les trafiquants (Misse, 2011, p. 18). La concurrence entre ces groupes criminels entraîne une prolifération d'actes de violence, une "course à l'armement " ainsi qu'un rapprochement entre criminels, policiers et autres pouvoirs publics corrompus.

11 En parallèle, les milices, ou paramilitaires, se multiplient, principalement à partir des années $1990^{2}$, composées par des policiers, pompiers, militaires, gardes pénitenciers et autres fonctionnaires actifs ou retraités. Ils justifient leur contrôle des territoires par la garantie de la sécurité des habitants en échange d'une taxe (souvent imposée ou extorquée aux habitants et commerçants). Ils détiennent d'autres services économiques informels tels que la télévision, internet, de l'immobilier ou encore des transports en commun. (Zaluar \& Siqueira Conceição, 2007). Face à la violence, au surarmement, aux liaisons avec l'appareil policier et politique, ainsi qu'aux conflits de territoires avec les factions, les milices ne garantissent pas une plus grande sécurité pour ces localités. Le Jogo de Bicho ayant perdu son influence à partir des années 1980, les territoires se répartissent entre les trafiquants ( $\mathrm{CV}: 31,4 \%$, ADA : 13,9\%, TCP : 11,8\%), les milices $(39,2 \%)$ et les pouvoirs publics (3,7 \%) (Misse, 2011, p. 19). 


\section{La domination locale des trafiquants}

12 La domination des trafiquants prend différentes formes, dans certains cas l'autoritarisme du chef s'affirme car il est une personnalité importante et connue de la communauté. Certains tentent de légitimer leur pouvoir en participant à des actions philanthropiques, telles que l'amélioration des équipements, l'aide aux plus démunis ou bien par l'offre de loisirs (bals de samba, pagode, funk,...). D'autres assoient leur pouvoir sur une tyrannie centralisée, où le chef, souvent non natif de la favela, s'impose par la peur (Misse, 2008 ; Zaluar,1998, 2000, 2004). A l'aune des théories de la formation subjective de l'ethos guerrier de Norbert Elias (1974), Alba Zaluar parle d'« ethos de la masculinité dominante, insoumise et provocatrice » (Zaluar, 2009, p. 15). Selon elle « les pratiques du monde du crime sont liées à un ethos de masculinité exacerbé, exagéré, centré sur l'idée d'un chef despotique dont les ordres ne pourraient pas être désobéis » (Zaluar, 2009, p. 575). Selon les favelas et le mode de domination des groupes criminels on observe une distinction entre le «bandit social» et le "méchant bandit ». Licia Valladares souligne que certains habitants des favelas craignent plus la police que les trafiquants et attribuent une morale particulière aux bandits: celle de l'honneur (Valladares, 2006, p. 173). Les trafiquants deviennent pour certains jeunes un référentiel d'une identité locale. Dans la favela de Rocinha l'ancien chef des trafiquants de la faction ADA était reconnu pour ses actions philanthropiques auprès d'une partie de la communauté.

« Nem pratiquait le banditisme social. À Noël, il braquait un camion de Sadia [une entreprise de production alimentaire] et distribuait la marchandise à tout le monde. C'est comme ça que Nem faisait. Nem parlait de lutte de classes, il parlait de division du travail, [...] il ne voulait pas de conflit avec la police. Selon les informations, une bonne partie de son argent était pour payer la corruption, parce qu'il ne voulait pas de conflit avec la police. [...] Il était très intelligent. Il réfléchissait sur la question du banditisme social. Tout le monde l'adore. On l'appelait le maître de la communauté » (Lieutenant de l'UPP de Rocinha).

13 Toutefois, ce type de réflexions risque de renforcer l'idée que les habitants seraient de connivence avec les trafiquants. Or, sans ignorer pour autant que le partage d'un même territoire induit des rapprochements de divers ordres (relations de voisinage, de parenté, liens économiques), il s'agit plutôt d'une complicité contrainte et non désirée, soumise à la loi du trafic et du silence. Certaines actions pouvant apparaitre comme de la bienfaisance peuvent cacher les intérêts propres des GCA, c'est-à-dire leur objectif de contrôle du territoire et de sa population pour donner libre cours aux trafics.

«Les trafiquants faisaient semblant de vouloir nous aider pour garder le contrôle.

Ils m'ont souvent proposé de l'argent, de me donner des ordinateurs, etc. Moi, je n'accepte pas » (Leader d'une ONG et habitant de Rocinha).

«Les trafiquants ne se préoccupent pas des projets sociaux, évidemment, ils jouent le rôle de la police parce qu'elle n'était pas présente! [...] Si tu allais voir un trafiquant pour demander de l'aide, oui, il pouvait t'aider, mais il n'y avait rien d'organisé, c'était vraiment des actions ponctuelles. Ils n'ont jamais eu de projets, les seuls projets ici, c'est le gouvernement, les ONG ou les églises » (Leader de l'association des habitants à Rocinha et habitant).

14 L'organisation des trafiquants autour des bocas de fumo (points de vente de drogue) est une organisation autonome, différente de celle des habitants. La notion même d'organisation peut être remise en cause. Selon Michel Misse, le crime organisé prend la forme « d'organisation, réseau ou groupe qui incorpore la violence comme ressource régulière de ses actions" (Misse, 2011, p.15). Bien qu'il y ait une hiérarchie des 
pouvoirs et une division du travail entre les trafiquants (le chef, les différents gérants : cannabis, cocaïne, sécurité, les petites mains, etc.), Michel Misse parle de groupes fragmentés et vulnérables. S'il s'agit de réseaux, il n'y a pas pour autant une organisation qui les coordonne et la méfiance entre les factions est très importante. Ainsi l'organisation, si elle existe, reste limitée, le trafic «n'est pas directement subordonné, ni à d'importants grossistes, ni à des organisations verticales de type familial ou patrimonial, à l'instar des cartels ou des mafias. » (Misse, 2008, p. 497) et ne se base pas sur les principes de l'honneur et de la fidélité (Machado da Silva, 2008). Le trafic ne possède donc pas nécessairement cette caractéristique structurante dans les communautés (Soares Gonçalves, 2010). Il pourrait plus s'agir de "coopératives criminelles » qui ne correspondraient pas à un pouvoir parallèle, car elles n'ont pas de projet politique alternatif (Lopes de Souza, 1996, p. 36).

Les trafiquants sont donc responsables d'un ordre social qui soumet les habitants: Louis Antonio Machado da Silva parle de «sociabilité violente» (Machado da Silva, 2008). Toutefois, si la sociabilité, comme le font justement remarquer A. Zaluar et A. P. Alles Ribeiro (2009) au regard de la définition de Simmel (1917), est «la forme d'existence sociale libérée de tout contenu social, affranchie de toute motivation individuelle pour n'apparaitre que comme impulsion, valeur en soi ", elle ne peut être violente car la violence est un moyen pour atteindre des fins matérielles, politiques ou symboliques. Alba Zaluar préfère l'usage « d'éthos guerrier » voire de "capital social négatif » qui serait en quelque sorte un capital destructeur de civilité pesant sur l'organisation sociale de la favela et détruisant de façon violente les réseaux horizontaux locaux (Zaluar \& Alles Ribeiro, 2009). Le terme "capital social négatif " peut, néanmoins, apparaitre contradictoire suivant que l'on se place du point de vue des trafiquants ou des habitants. Inévitablement, cet ordre violent fragilise les relations et le lien social entre les habitants en générant un manque de confiance envers le voisinage ainsi que la peur de la dénonciation et des représailles ; ce qui rend difficile la constitution d'une base pour l'action collective. Les relations sociales sont ainsi structurées par l'usage d'une force privatisée qui nourrit la violence urbaine.

\section{L'héritage de la dictature : une police violente, arbitraire et corrompue}

16 A Rio de Janeiro, le paradoxe de l'institution policière se trouve dans une police qui d'une part réprime fortement la criminalité, parfois sans distinction entre habitants de favela et criminels, et qui d'autre part tire des bénéfices des marchés illégaux grâce à la corruption. Les habitants sont alors presque systématiquement pris entre deux forces : celle de la police et celle des groupes de trafiquants. De plus, le climat de peur et la stigmatisation des habitants des favelas inhibent les organisations collectives locales susceptibles de dénoncer l'un ou l'autre type d'abus de pouvoir. À Rio de Janeiro, l'usage de la violence, les abus d'autorité et l'irrespect des droits sont des attitudes fréquentes de la part des policiers (contrôle d'identité et fouilles violentes, arrestations arbitraires, moyens mis en œuvre disproportionnés, usage d'armes, recours à des pratiques vexatoires,...). Comme le souligne Alba Zaluar, la police, au Brésil, est imprégnée de la mentalité du régime militaire et basée sur l'idée d'un ennemi intérieur à éliminer (Zaluar, 2004). Cette forme de violence policière a bénéficié d'une certaine impunité de la part d'une partie du public plus aisé, les exécutions extrajudiciaires étant parfois perçues comme le meilleur moyen de se débarrasser des criminels, face à 
un système judiciaire considéré comme défectueux. La violence institutionnelle au Brésil passe par un « usage illégal, illégitime et indu de la force par l'appareil répressif d'un État » (Daudelin, 1996, p. 97).

\section{Une superposition des espaces de criminalité, d'insécurité et d'intervention?}

\section{Insécurité réelle vs insécurité perçue}

17 À Rio de Janeiro, les morts violentes ${ }^{3}$ ont diminuée de $35 \%$ entre 2001 et 2012, les vols sans violence et vols avec usage de la violence ont respectivement augmenté de $82 \%$ et de 5,5\%. Entre 2007 et 2012, tandis que les homicides ont baissé de $34 \%$ et les actes de résistance de $64 \%$, le nombre de personnes disparues a augmenté de $28 \%$, tout comme les menaces $(+32 \%)$ et les viols $(+338 \%)^{4}$. Tout en ayant une définition à la fois légale et sociale, la violence urbaine est également une représentation sociale, distinguant l'insécurité réelle (les statistiques) du sentiment d'insécurité. Dans les sociétés modernes «l'exaspération du souci sécuritaire engendre nécessairement sa propre frustration, qui nourrit le sentiment d'insécurité » (Castel, 2003, p. 23). À Rio de Janeiro, en enregistre une "sursécurisation » des lieux : les caméras de surveillance prolifèrent, les véhicules sont encore autorisés à ne pas s'arrêter aux feux rouges la nuit en raison de risque d'agression, les condominiums fermés et ultra surveillés se multiplient etc. Cette surprotection renforce l'insécurité perçue, alimentée également par les médias. Par ailleurs, le contexte de militarisation de la police et le caractère spectaculaire des opérations de pacification n'engendrent-ils pas à nouveau une catégorisation des favelas comme lieux de danger par excellence? Dans quelle mesure les favelas sontelles des territoires plus dangereux que le reste de la ville?

Si les habitants des zones aisées ont tendance à stigmatiser les favelas comme lieu d'insécurité, au contraire, les habitants des favelas associent souvent une forme de sécurité intrinsèque à la communauté. Les crimes auraient notamment lieu en dehors car les trafiquants puniraient sévèrement de tels actes. Dans ce cas, la frontière de la favela marque un périmètre de protection au sein duquel les habitants atteignent une forme de « sécurité communautaire ». Concernant les morts violentes, il serait hâtif de conclure à leur surreprésentation systématique dans les favelas par rapport aux autres quartiers plus aisés de la ville. En effet, au regard de la cartographie des homicides dans la zone sud de Rio de Janeiro (2006-2009) on observe plus d'homicides hors des limites des favelas (cf. illustration 4). 
Illustration 4 - Cartographie des homicides dans la zone sud de Rio de Janeiro

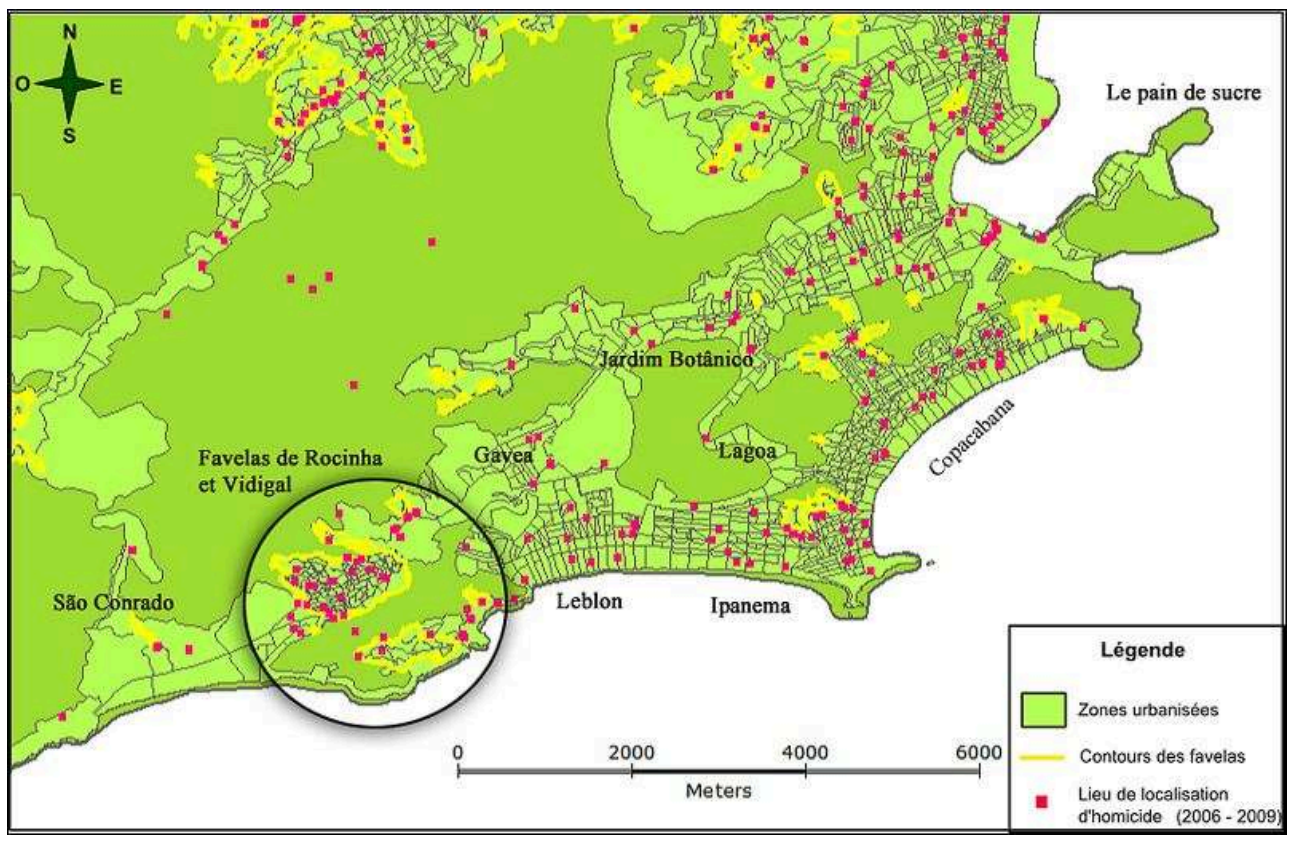

Sources : Fiocruz, ISP

19 Il faut cependant relativiser cette localisation des actes criminels. En effet la méconnaissance des structures spatiales de la favela, notamment des noms de rues, pourrait amener à recenser certains crimes en dehors ou à l'entrée des favelas alors qu'ils ont eu lieu à l'intérieur. Sur 38 homicides ayant eu lieu entre 2008 et 2012 dans l'AISP 23 (quartiers d'Ipanema, Leblon, Gávea, Jardim Botânico, Lagoa, Rocinha, São Conrado et Vidigal : cf. illustration $\left.{ }^{\circ} 3\right), 21$ ont eu lieu à Rocinha et Vidigal, soit $55 \%$ des homicides ${ }^{5}$. Or ces favelas ont été pacifiées en 2011. Nous pouvons nous interroger sur l'impact de la pacification sur le sentiment de sécurité des habitants? Remplacer le contrôle armé des trafiquants par celui de la police diminue-t-il le sentiment de soumission à un ordre imposé par la force?

\section{L'apport de la pacification dans le champ de la sécurité publique}

Alors que certains habitants émettent des réserves sur les politiques publiques de sécurité en raison de leur manque de confiance envers les institutions et la police, d'autres voient dans ce programme de pacification un espoir offrant une alternative à la terreur imposée par les trafiquants, les milices ou par des interventions policières ponctuelles et violentes. L'espoir allait parfois au-delà de ce que cette nouvelle politique proposait réellement. Ainsi, certains pensaient qu'elle ferait disparaître le trafic et réduirait radicalement la criminalité. Or la pacification ne prétend pas anéantir le narcotrafic, elle a pour objectif le démantèlement des GCA et la reprise de contrôle des territoires. On peut donc examiner l'apport de la pacification des favelas dans le champ de la sécurité publique. D'une part, la pacification permet de rétablir les droits fondamentaux des habitants des favelas, qui étaient auparavant limités par les groupes criminels présents: le droit à la vie, le droit d'aller et venir, le droit de propriété, l'accès à la justice, à la santé, aux équipements et services collectifs. D'autre part, les habitants subissent moins au quotidien la peur des conflits entre factions 
opposées ou entre trafiquants et policiers, et les risques engendrés par les échanges de tirs et les balles perdues (Leite, 2013). On observe une évolution des crimes recensés dans les favelas pacifiées ${ }^{6}$ diminution des morts violentes et plus particulièrement de celles liées aux interventions policières, tout comme des vols avec usage de la violence. Inversement, les agressions, menaces et vols sans violences ont crû significativement (Cano, 2012). Ces augmentations peuvent être liées à l'effritement de la culture de la peur et de la loi du silence, qui n'empêche pas certains de commettre des délits mais permet, inversement, à d'autres de les dénoncer plus facilement en raison d'une justice plus accessible. Ainsi, à Vidigal, presque tous les indices criminels ont augmenté entre 2007 et 2013 : les victimes de crimes violents $(+265 \%)$, dont les dommages corporels (+314\%), les menaces $(+208 \%)$, les vols violents $(+100 \%)$, les vols $(+262 \%)$ et enfin globalement, les registres d'occurrence ont été multiplié par 2,57.

«Avant il existait la peur du jugement du trafiquant. Celui qui avait volé on lui coupait la main ; si deux femmes se battaient: on leur rasait les cheveux. Les gens avaient peur du jugement des trafiquants, ils savaient qu'il y avait des représailles physiques, voire vitales. Aujourd'hui celui qui vole est emprisonné, certains arrivent à fuir. Donc oui, les vols vont augmenter, c'est sûr. Les lésions corporelles ont aussi augmenté. Avant la femme allait se plaindre au trafiquant : « mon mari est saoul, il veut me battre » et le trafiquant pointait son arme sur la tête du mari. Aujourd'hui, la femme doit venir porter plainte au poste donc forcément les statistiques augmentent. Avant, les choses arrivaient et la police ne savait pas » (Lieutenant de l'UPP de Rocinha).

La police tente aujourd'hui de prendre un nouveau visage en se rapprochant de la population et en étant présente quotidiennement. Comment s'organisent ces nouveaux acteurs de la gouvernance locale?

\section{De nouvelles règles pour les habitants et de nouvelles pratiques pour la police}

\section{La police communautaire}




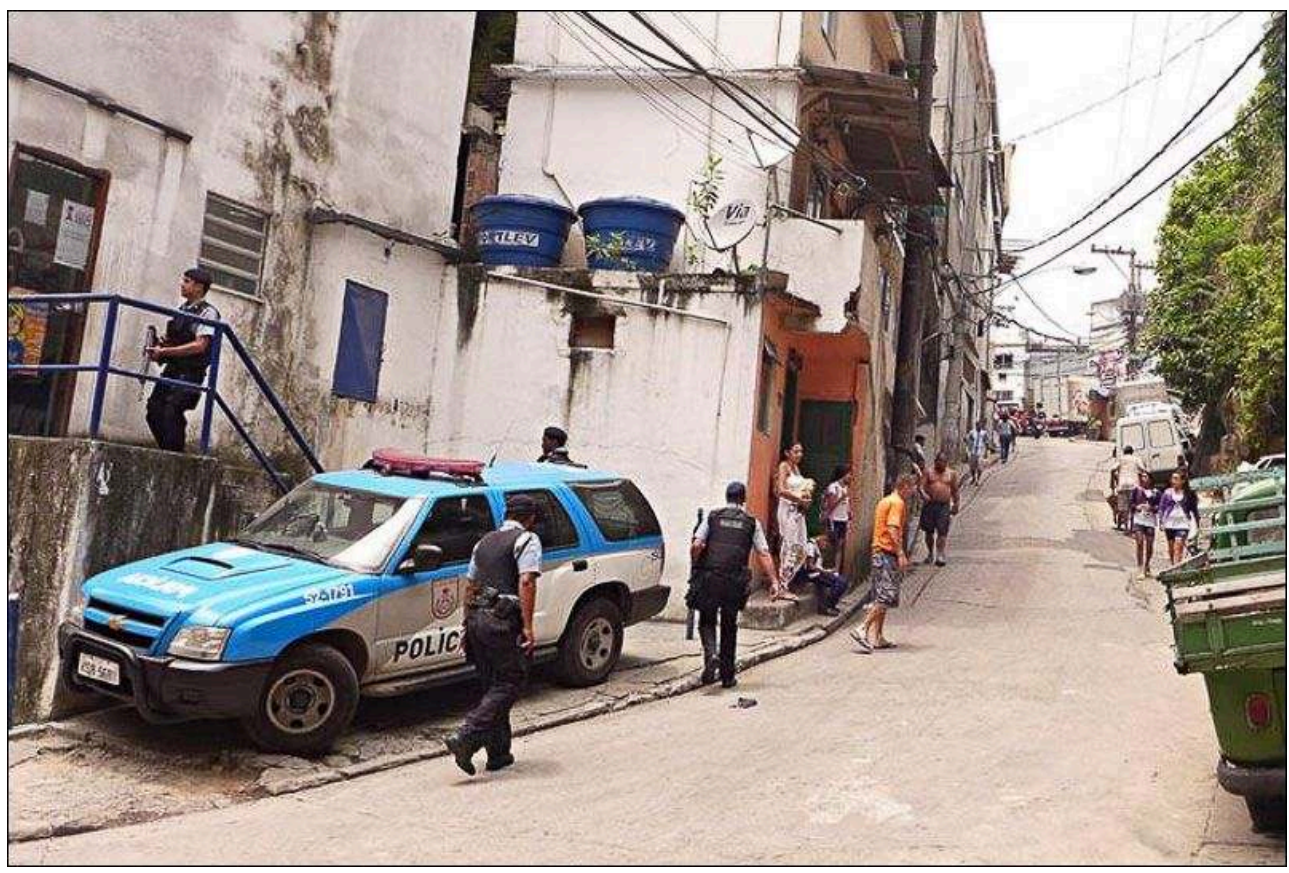

Poste UPP situé sur la route principale de Vidigal, voiture de patrouille et policiers.

Face à une nécessaire réforme des polices et du système pénal l'État s'inspire des modèles de polices communautaires des USA. Dans une philosophie d'intégration et d'interaction entre les favelas et les autres quartiers, le Brésil crée sa propre «police communautaire » en 2007 (au Mato Grosso, Ceará, Pará et à Rio de Janeiro où elle prend le nom d'UPP). Pour le Ministère de la Justice « les institutions de l'État et la population locale doivent travailler ensemble pour identifier, hiérarchiser et résoudre les problèmes qui affectent la sécurité publique, telles que la criminalité, la peur, l'exclusion sociale et l'inégalité qui accentuent les problèmes de criminalité et entravent la possibilité d'améliorer la qualité de vie des citoyens». La police communautaire associe la prévention du crime à l'identification et la résolution des problèmes de défense sociale avec la participation de la communauté. Les policiers sont engagés jeunes afin d'éviter les anciennes pratiques corrompues, ils reçoivent une prime de $500 \mathrm{R} \$$ ainsi qu'une formation spécifique sur les principes de la police communautaire ${ }^{8}$. Observe-t-on pour autant un changement dans les pratiques? Cette nouvelle police ne renforce-t-elle pas les clivages sociaux en intégrant la logique traditionnelle de politiques publiques distinctes à l'égard des favelas? En rappelant que derrière le terme police communautaire ou police de pacification on parle de «police de favela ».

« Nous avons connu une police de guerre, une police tournée vers le combat, vers la répression. Et aujourd'hui, on essaye de changer cette police: une police plus tournée vers les droits humains, plus formée pour répondre aux besoins élémentaires de la population. La police est aujourd'hui plus qualifiée » (Lieutenant de l'UPP de Rocinha).

23 Cette nouvelle présence policière quotidienne demande aux habitants aux habitants de s'adapter à de nouvelles règles et pratiques sociales dans la favela qui peuvent susciter au départ une certaine résistance. Il s'agit d'une forme de "choc de l'ordre » où des obligations et des règles sont soudainement imposées à une communauté qui vivait 
avec ses propres règles. Les habitants doivent s'ajuster à cette nouvelle réalité où ce qui avant était résolu par les groupes criminels l'est aujourd'hui par la police.

«La communauté a passé beaucoup de temps sans policiers, le policier était celui qui venait pour tuer, pour tirer. Quand les policiers arrivaient, il fallait fermer les portes et les fenêtres et rester à la maison. Aujourd'hui, ils peuvent compter sur les policiers. C'est une police beaucoup plus transparente et tournée vers les nécessités de la communauté » (Lieutenant de l'UPP de Rocinha).

24 Les policiers multiplient les rôles dans la favela : détenteurs de la coercition légitime, médiateurs de conflits, professeurs de sport, animateurs radio etc. Cependant, la multiplication des fonctions des policiers dans la favela ne risque-t-elle pas de désorienter la population quant au comportement à adopter face à ces policiers ? Comment allier le pouvoir de violence légitime et le rôle d'éducateur?

« À l'association des habitants, il y a un policier qui donne des cours de judo. Il n'est pas armé mais en contre-partie il y a deux policiers dans la salle qui sont armés pour surveiller » (Habitant de Vidigal).

25 L'UPP de Rocinha a mis en place, début 2013, un service clients sous forme de centre d'appel pour connaitre l'avis des habitants sur le comportement des policiers (les agents de l'UPP appellent les habitants pour poser des questions sur la qualité du service, le comportement du policier et leur satisfaction lors de l'intervention).

\section{D'anciennes pratiques policières qui persistent}

Une partie de la population dénonce l'attitude de policiers qui semblent plus préparés à affronter les trafiquants qu'à collaborer avec les habitants, souvent traités comme des suspects. Les violences policières sont encore courantes, tout comme le non-respect de la propriété.

« La police n'a pas la capacité de maintenir toute la communauté en paix. Parce que même avec l'UPP, le crime et la drogue existent, il existe encore tout ce que l'extérieur croit qu'il n'y a plus depuis la pacification. Ils disent : Rocinha c'est bien ! C'est bien en rien! Il y a tout le temps de la violence et des bagarres. Rocinha est pire! Ils font soit disant des projets pour améliorer Rocinha. Ils n'améliorent rien, au point qu'on tire encore sur des touristes ici'![...] Lors de la pacification ils sont entrés dans mon atelier pour chercher des armes ou de la drogue. Ils ont tout cassé, ils ont cassé des projets d'art que j'avais mis un temps fou à réaliser. Ils ont donné des coups de pied dans mes maquettes. [...] Comment peut-on faire avancer les choses en désarmant les trafiquants pour que finalement la police prenne le contrôle de la favela et soit pire que les trafiquants? Ils ne respectent pas les habitants, ils les frappent. Pour moi, l'UPP c'est une façade jusqu'à ce que la Coupe $\mathrm{du}$ Monde se termine [...] les favelas n'ont pas avancé avec les UPP » (Leader d'une ONG et habitant de Rocinha).

«L'excès est une contre réponse de la résistance à l'interpellation de la part des habitants. Certains attaquent la police, alors on doit faire un usage progressif de la force. [...]Les gens ne savent pas comment se comporter quand ils sont contrôlés » (Lieutenant de l'UPP de Rocinha).

En 2013, suite à la dénonciation de corruption, d'abus de pouvoir et même de torture envers les habitants ainsi que de la reprise de confrontations armées dans des favelas pacifiées, notamment à Rocinha, l'image des UPP est entachée. Lors de mes enquêtes de terrain à Vidigal, des témoignages évoquent l'activité de points de vente de drogue sous couvert d'agents de l'UPP, ainsi que les gratifications illégales que recevraient des policiers gradés en échange de leur autorisation pour la réalisation de soirées payantes. 
En octobre 2013, une dizaine de policiers de l'UPP de Rocinha, dont le comandantmajor, étaient emprisonnés pour séquestration suivie de mort d'un habitant de Rocinha: Amarildo. Ce fait divers confirme à la fois la perpétuation de pratiques violentes et abusives des policiers et la confusion encore trop systématique entre habitant de favela et bandit. Mais dorénavant l'action collective se renforce avec une mobilisation des habitants qui font entendre leur voix en dépassant la « loi du silence ». En effet, les cas semblables n'étaient pas rares avant la pacification, mais le débat n'était pas porté sur l'espace public. Avec les promesses de sécurisation du gouverneur de l'État de Rio Sergio Cabral et du maire Edouardo Pães, les classes moyennes, les intellectuels et les médias se font le relais des revendications des classes populaires déjà plusieurs fois lésées par des tentatives avortées d'amélioration des conditions de vie et de sécurité dans les favelas. Dans tout le Brésil, les méthodes répressives de la police militaire ont été fortement décriées lors des manifestations qui ont lieu depuis juin 2013. Le thème de la violence policière sort alors du contexte des favelas. Les brésiliens s'interrogent sur la capacité des forces de l'ordre à contenir en douceur les débordements, que ce soit pour les mobilisations actuelles ou pour des attroupements futurs lors de la Coupe du Monde de 2014 par exemple. Parallèlement, les trafiquants profiteraient de ce climat de tension et de fragilisation de l'image de l'UPP pour déstabiliser cette occupation en reprenant les confrontations entre factions ennemies, plus particulièrement à Rocinha.

\section{Conclusion}

Afin d'éradiquer le contrôle des favelas par les GCA, les autorités publiques ont redéfini leur politique de sécurité en la territorialisant. Ces espaces nécessitent une action spécifique et adaptée, mobilisant des moyens considérables afin qu'ils s'intègrent au reste de la ville. La présence quotidienne de policiers intensifie leur rôle dans la médiation de conflits et contribue à une meilleure accessibilité des lieux. Bien que certains bandits soient encore présents, ainsi que des points de ventes de drogue moins visibles, la pacification a permis la disparition du contrôle ostensiblement armé des groupes criminels dans les favelas ciblées. Le bilan à court terme de la pacification est toutefois mitigé notamment face à la perpétuation d'abus d'autorité de la part de policiers. La multiplication des rôles qui leur sont attribués pourrait dissimuler une concentration excessive de pouvoir et maintenir les habitants sous une forme nouvelle de domination.

Ces nouvelles orientations posent question face à l'exigence d'égalité entre les citoyens, puisque la territorialisation de l'action publique implique qu'une même politique puisse prendre des inflexions différentes suivant les espaces et les populations auxquels elle s'applique. Les résultats ne sont pas similaires entre les favelas pacifiées, ce qui souligne à nouveau la spécificité de chacune d'elles. Par exemple, si généralement les risques quotidiens d'échanges de tirs ont disparu, ce n'est pas le cas à Rocinha. On peut se demander si la territorialisation contribue à résorber les inégalités sociales ou si au contraire, elle accroît les disparités territoriales. On observe en effet une hiérarchisation des espaces où l'on souhaite réinstaurer l'ordre : le choix des favelas ne correspond pas nécessairement à des situations d'urgence liées à l'insécurité ou à la violence, les quartiers sécurisés sont des lieux stratégiques et symboliques de la ville (Bautes et Soares Gonçalves, 2011). La pacification a des limites géographiques 
puisqu'elle concerne d'abord les favelas de la zone sud, c'est-à-dire celles qui ont le plus fort potentiel économique et touristique, avant de s'attaquer à celles de la zone nord, la zone ouest étant encore très peu touchée par cette politique (les favelas de cette zone sont principalement sous contrôle des milices). Pour l'instant, une seule favela tenue par les milices a été pacifiée, alors que les milices dominent la majorité des favelas à Rio de Janeiro ; sur 997 favelas, 166 ont été pacifiées, 455 sont encore dominées par des milices et 362 par les trafiquants ${ }^{10}$.

En conclusion, soulignons brièvement les conséquences indirectes de la pacification. "L'effet UPP » a notamment contribué à accentuer la spéculation immobilière dans les favelas de la zone sud, obligeant une partie des habitants les plus vulnérables à quitter leur lieu de vie pour s'installer plus loin en périphérie. Nous pouvons prendre ici l'exemple de Vidigal, où le processus de "classe-moyennisation » déjà impulsé depuis quelques années s'est renforcé avec l'arrivée des UPP. La perspective de plus-value des terrains pousse les classes plus aisées à investir ainsi que certains habitants prenant part à cette nouvelle économie de marché, tandis que d'autres n'arrivent pas à suivre l'augmentation du coût de la vie, principalement les locataires ${ }^{11}$, notamment en raison du coût des services lié à la régularisation de l'espace (électricité, eau ${ }^{12}$, impôt sur la propriété du sol,...). Ce phénomène d'éviction implicite renforce alors la redéfinition du quadrillage socio-spatial de la ville avec une périphérisation de la pauvreté de plus en plus marquée (et de la violence, une partie des criminels ayant fui vers ces périphéries). Phénomène auquel contribuent, entre autres, les opérations de relogement en périphérie des familles dont les habitations sont détruites, le plus souvent dans les favelas, au profit de la construction d'équipements publics ou dans le cadre des travaux d'aménagement de la Coupe du Monde et des Jeux Olympique. Finalement, la pacification s'inscrit dans des orientations plus larges de sécurisation et d'affirmation de la ville de Rio de Janeiro comme ville globale. Qu'en sera-t-il de la pérennité de cette politique au lendemain des méga-événements? Va-t-elle s'étendre aux favelas situées plus en périphérie?

\section{BIBLIOGRAPHIE}

Bautes N. \& Soares Gonçalves R., 2011. Sécuriser l'espace des pauvres. Sécurité publique et justice spatiale dans les favelas de Rio de Janeiro. Justice Spatiale/Spatial Justice. Pratiques de Sécurité en Ville (coord. J. Tadié \& M. Morelle), $\mathrm{n}^{\circ} 4$.

Cano I., 2012. Os Novos Donos do Morro. Rio de Janeiro, Pesquisa LAV, 227 p.

Castel R., 2003. L'Insécurité sociale : qu'est-ce qu'être protégé ? Paris, Seuil, 95 p.

Daudelin, J., 1996. Making sense of evil: the politics of institutional violence in Brazil. Canadian journal of development studies, $\mathrm{n}^{\circ}$ spécial, p. 93-111.

Elias N., 1974. La civilisation des mœurs. Paris, Pocket, 342 p. 
Leeds E., 1998. Cocaína e poderes paralelos na periferia urbana brasileira, améaças à democratização em nivel. In Zaluar A. \& Alvito M., Um seculo de favela, Rio de Janeiro, FGV, p. 231-276.

Misse M., 2008. Le Movimento. Les rapports complexes entre trafic, police et favelas à Rio de Janeiro. Déviance et Société, vol. 32, n4, p. 495-506.

Misse M., 2011. Crime organizado e crime comum no rio de janeiro: diferenças e afinidades. Dossiê "crime, segurança e instituições estatais: problemas e perspectivas. Revista de sociologia e política, vol. 19, n40, p. 13-25.

Soares Gonçalves R., 2010. Les favelas de Rio de Janeiro : Histoire et droit, XIX et XX $X^{e}$ siècles. Paris, L'Harmattan, Recherches Amériques latines, 295 p.

Valladares L. de P., 2006. La favela, d'un siècle à l'autre. Édition la maison des sciences de l'homme, $230 \mathrm{p}$.

Waiselfisz, J. J. (2013). Mapa da violencia 2013 Homicídios e Juventude no Brasil. Brasilia: SecretariaGeral da Presidência da República, 100p.

Zaluar, A. (. (2007). Pesquisa de vitimização do Rio de Janeiro, relatório técnico. Rio de Janeiro: NUPEVI/ UERJ.

Zaluar, A. (1998). Crime, medo e politica. Dans Zaluar A. \& Alvito M., Um século de favela. Rio de Janeiro: FGV (pp. 209-232)

Zaluar, A. (2004). Integração Perversa: pobreza e tráfico de drogas. Rio de Janeiro: FGV, 438p.

Zaluar, A., \& Siqueira Conceição, I. (2007). Favelas sob o controle das Milícias : que paz? São Paulo em Perspectiva, v.21, $n^{\circ} 2$, pp. (89-101).

Zaluar, A. (2009, octobre). Agressão fisica e gênero na cidade do Rio. Agressão física e gênero na cidade do Rio de Janeiro., v.24, n71, (pp. 9-24).

Zaluar, A. (2009, octobre). Pesquisando no perigo: ethnografias voluntarias e não acidentais. Mana, $n^{\circ} 15$, (pp. 557-584).

Zaluar, A., \& Alles Ribeiro, A. P. (2009, Juillet). Teoria da eficacia coletiva e violência : o paradoxo do suburbio carioca. Novos Estudos CEBRAP, (pp. 175-196).

\section{NOTES}

1. Il s'agit d'entretiens et observations de terrain lors d'un séjour de recherche de septembre 2012 à juin 2013 dans le cadre d'un doctorat. J'ai privilégié une approche ethnographique notamment sous forme d'observation participante en logeant à Vidigal et en participant à différentes activités associatives dans cette même favela et à Rocinha. Cette recherche mobilise à ce jour une dizaine d'entretiens formels et de nombreux entretiens informels avec, entres autres, des leaders communautaires, des habitants ou des policiers.

2. On distingue plusieurs types de «milices ». Elles existaient déjà dans les années 1950 allant de l'offre de protection aux commerçants et habitants, à une organisation presque de type mafieuse avec des ambitions politiques où la protection passe en partie par l'association des habitants, exemple de la favela Rio das Pedras située dans la zone ouest de Rio de Janeiro (Misse, 2011).

3. Selon les statistiques de l'Institut de Sécurité Publique (ISP), les morts violentes comprennent les victimes d'assassinat, de vol à main armée, d'agression physique et d'acte de résistance : mort liée à une intervention policière.

4. ISP (2007-2012) 
5. ISP

6. Résultat sur les 13 premières UPP sur les chiffres de 2006-2011

7. ISP

8. Ministère de la justice : http://portal.mj.gov.br

9. En mai 2013, quelques jours avant mon entretien avec ce responsable d'une ONG, un touriste allemand se faisait tirer dessus par un trafiquant à Rocinha ; il sera seulement blessé.

10. Groupe de recherche du NUPEVI sous la direction d'Alba Zaluar, UERJ, mai 2013.

11. Les prix ont presque doublé, par exemple le loyer d'un studio est passé de $300 \mathrm{R} \$$ avant la pacification à $600 \mathrm{R} \$$.

12. Auparavant, les habitants étaient branchés illégalement sur les réseaux et ne payaient donc pas de facture.

\section{RÉSUMÉS}

La sécurité publique est un défi posé au Brésil et plus particulièrement à Rio de Janeiro. La ville, qui cherche à s'inscrire comme acteur central sur la scène internationale, a redéfini sa stratégie sécuritaire en réaffirmant le pouvoir des autorités publiques dans les favelas grâce à la «policiarisation» et à des politiques publiques territorialisées. Longtemps stigmatisées et délaissées par les pouvoirs publics, les favelas ont dérivé sous le contrôle de groupes criminels armés. À travers la mise en place d'une police de proximité depuis 2008, la pacification des favelas entreprend de reconstruire une police plus compétente, plus transparente et moins répressive.

Public security is a challenge in Brazil, especially in Rio de Janeiro. The city, which seeks to be a central player on the international scene has redefined its security strategy by reaffirming the public authorities power in the favelas through "militarization" and territorialised policy. The favelas which have been stigmatized and abandoned by the government, have drifted under the control of armed criminal groups. Through the establishment of a community policing for 2008, the pacification of the favelas began to rebuild a more competent, more transparent and less repressive police.

\section{INDEX}

Mots-clés : politique de sécurité publique, police communautaire, territorialisation, favela, groupe criminel

Keywords : public security policy, community policing, territorialisation, favela, criminal group

\section{AUTEUR}

\section{JUSTINE NINNIN}

Justine Ninnin, justine.ninnin@neuf.fr, est doctorante à l'École Doctorale de Géographie de Paris et à l'Université d'État de Rio de Janeiro. Elle a publié récemment :

Justine Ninnin, « Le rêve carioca : entre planification urbaine et déplacements forcés de 
population ", L'Espace Politique [En ligne], 22 | 2014-1, mis en ligne le 18 mars 2014. URL : http:// espacepolitique.revues.org/2950 ; DOI : 10.4000/espacepolitique.2950 